\title{
Acetylcholine Receptors Direct Rapsyn Clusters to the Neuromuscular Synapse in Zebrafish
}

\author{
Fumihito Ono, ${ }^{1,2}$ Gail Mandel, ${ }^{3,4}$ and Paul Brehm ${ }^{3}$ \\ ${ }^{1}$ The Whitney Laboratory, University of Florida, St. Augustine, Florida 32080, ${ }^{2}$ Department of Neuroscience, University of Florida, Gainesville, Florida \\ 32610, and ${ }^{3}$ Department of Neurobiology and Behavior and ${ }^{4}$ Howard Hughes Medical Institute, State University of New York at Stony Brook, Stony Brook, \\ New York 11794
}

Clustering of nicotinic muscle acetylcholine receptors (AChRs) requires association with intracellular rapsyn, a protein with an intrinsic ability to self-cluster. Previous studies on sofa potato ( $s$ p), an AChR null line of zebrafish, have suggested that AChRs may play an active role in subsynaptic localization of rapsyn clusters. To test this proposal directly, we identified and cloned the gene responsible for the sop phenotype and then attempted to rescue subsynaptic localization of the receptor-rapsyn complex in mutant fish. sop contains a leucine to proline mutation at position 28, near the N terminus of the zebrafish AChR $\delta$ subunit. Transient expression of mutant $\delta$ subunit in sop fish was unable to restore surface expression of muscle AChRs. In contrast, expression of wild-type $\delta$ subunit restored the ability of muscle to assemble surface receptors along with the ability of fish to swim. Most importantly, the ability of rapsyn clusters to localize effectively to subsynaptic sites also was rescued in large part. Our results point to direct involvement of the AChR molecule in restricting receptor-rapsyn clusters to the synapse.

Key words: sofa potato; mutant; myasthenia; neuromuscular disease; synapse development; movement

\section{Introduction}

Zebrafish mutated with ethylnitrosourea have permitted identification of a large number of mutant lines with defects in swimming behavior (Granato et al., 1996). The defects range from complete paralysis to exaggerated motility with touch. The genetic bases for some of these mutants are beginning to be identified, and, not unexpectedly, many of the mutations affect the motor pathway. The paralytic mutants nic-1 (Westerfield et al., 1990) and sop (Ono et al., 2001), for example, lack muscle acetylcholine receptors (AChRs), whereas the paralytic line relaxed lacks functional muscle dihydropyridine receptors (Ono et al., 2001). The mutant twitch once shows weak and abbreviated swimming in response to touch because of a mutation in muscle rapsyn (Ono et al., 2002). Mutations in the same domain of human rapsyn recently were shown to underlie congenital forms of myasthenic syndrome (Ohno et al., 2002). Another prevalent defect in swimming is discoordinated and synchronous contractions of opposing muscles with touch. In two such lines this has been shown to result from prolonged synaptic current decay pro-

\footnotetext{
Received March 9, 2004; revised May 10, 2004; accepted May 11, 2004.

This study was supported by Grants NS-18205 and NS-045935 from the National Institutes of Health. G.M. is an investigator of the Howard Hughes Medical Institute. We thank Dr. Frohnhoefer (Max Planck Institute, Tuebingen, Germany) for providing sofa potato zebrafish. SV2 antibody developed by Dr. Kathleen Buckley was obtained from the Developmental Studies Hybridoma Bank developed under the auspices of the National Institute of Child Health and Human Development and maintained by the University of lowa. Rich Grady provided expert maintenance of the fish colony, and Dr. Hua Wen offered expertise in the preparation of figures. We thank Dr. Joe Fetcho for the use of his high speed camera. J. Speh and P. Speh helped in the preparation of figures.

Correspondence should be addressed to Dr. Fumihito Ono, The Whitney Laboratory, 95050 cean Shore Boulevard,

St. Augustine, FL 32080.E-mail: fono@whitney.ufl.edu.

DOI:10.1523/JNEUROSCI.0851-04.2004

Copyright $\odot 2004$ Society for Neuroscience $\quad$ 0270-6474/04/245475-07\$15.00/0
}

ducing sustained contractions on both sides of the tail. In the line ache slowed synaptic current results from the lack of muscle acetylcholinesterase (Behra et al., 2002), whereas in the twister line prolongation results from a mutation in the pore of the AChR $\alpha$ subunit (Lefebvre et al., 2004).

Several factors can be credited for the recent success in identifying so many mutations involving the neuromuscular junction in zebrafish. The unique opportunities for in vivo imaging and physiological analysis of mutant synapses, the result of transparent skin and unfettered access to the neuromuscular synapse, have played major roles. The greatest advantage, however, lies in the rapid ex utero development of larvae, permitting even null mutants to survive well past formation of synapses. Consequently, mutations that are lethal in mammals at early stages of development can be studied at mature synapses by using zebrafish. In the present study, we exploit this advantage through use of an AChR null line of fish for which there is no mammalian counterpart. This advantage affords the opportunity to test a previous proposal whereby AChRs play an active role in subsynaptic localization.

The functional relationships among the many postsynaptic proteins expressed at the neuromuscular junction remain the focus of intensive studies (Sanes and Lichtman, 2001). At the heart of postsynaptic organization lie AChR and rapsyn, for which mutual interactions as well as interactions with other synaptic proteins are only beginning to be understood. Progress has been hampered by the fact that, in mammals, total knock-out of AChRs is lethal early in development. Thus much of our information has come from heterologous expression systems in which synapses are lacking. In a previous study, we showed that sop represents an AChR null mutant line of zebrafish. In this study, 
we found, quite unexpectedly, that rapsyn clusters failed to localize effectively to the synapses in living fish (Ono et al., 2001). This observation raised the possibility that the AChR was more than a passive player in the process of organizing the synapse and may actively navigate rapsyn clusters. However, without knowledge of the mutation in sop, it was not possible to draw firm conclusions. For example, a gene other than AChR could be mutated, and this gene product could direct rapsyn positioning and AChR expression in a parallel manner. In this study, we demonstrate that the mutation in sop, resulting in the absence of AChRs, is a point mutation in the $\mathrm{N}$ terminus of the $\delta$ subunit of the AChR. Using this fish, we perform experiments to examine directly the role of AChR in synaptic localization of rapsyn.

\section{Materials and Methods}

Fish strains. The mutant line of zebrafish sop $t j^{19 d}$ was obtained from Max Planck Institute (Granato et al., 1996; Ono et al., 2001). Adult fish heterozygous for the sop mutation were crossed to generate homozygous embryos. Transgenic fish expressing rapsyn protein conjugated with green fluorescent protein (rapsyn-GFP) were crossed with sop fish to generate a sop heterozygous fish harboring a rapsyn-GFP transgene. Embryos inheriting the rapsyn-GFP gene from both parents were not studied because this results in a large amount of extrasynaptic rapsyn-GFP that complicates analyses.

Cloning of the acetylcholine receptor $\delta$ subunit gene. The Expressed Sequence Tag (EST) database was searched for sequences showing homologies to $\delta$ subunit genes of other species. Sequences encompassing the start and the end of the coding region were retrieved (dbEST identification: 1139897 and 1141774). Primers responding to the $5^{\prime}$-untranslated region (UTR) and $3^{\prime}$-UTR of the putative gene were used to amplify $\delta$ sequence with PCR from cDNA of 4-d-old zebrafish. The accession number for zebrafish $\delta$ subunit sequence is AB120372.

Imaging. The antibody staining by synaptic vesicle protein 2 (SV2; Developmental Studies Hybridoma Bank, Iowa City, IA) was performed on fish fixed overnight in $4 \%$ paraformaldehyde. After fixation the fish were washed for $5 \mathrm{~min}$, treated with $-20^{\circ} \mathrm{C}$ acetone for $7 \mathrm{~min}$, and washed for an additional $5 \mathrm{~min}$. Then the fish were placed in PBS containing $2 \%$ horse serum and $0.5 \%$ Triton X-100. After $30 \mathrm{~min}$ the fish were placed in PBS containing $0.5 \%$ Triton X-100 and the primary SV2 antibody $(1: 500)$ at $4^{\circ} \mathrm{C}$ for overnight. After being washed in PBS with $0.5 \%$ Triton X-100, the fish were treated with a goat anti-mouse Cy5conjugated secondary antibody (1:500; Molecular Probes, Eugene, OR) at $4^{\circ} \mathrm{C}$ overnight. Then the fish were washed in PBS with $0.5 \%$ Triton $\mathrm{X}-100$ and imaged using confocal microscopy. The labeling of ACh receptors with rhodamine- $\alpha$-bungarotoxin (rh- $\alpha$-Btx; Molecular Probes) was performed on both living and fixed fish. The procedure for labeling living fish was described previously (Ono et al., 2002).

All of the images were taken using a Zeiss LSM510 Meta confocal microscope. GFP signals were excited at $488 \mathrm{~nm}$, and the emission was bandpass filtered between 505 and $530 \mathrm{~nm}$. Rhodamine signals were excited at $544 \mathrm{~nm}$, and the emission was bandpass filtered between 560 and $610 \mathrm{~nm}$. Cy5 signals were excited at $633 \mathrm{~nm}$, and the emission was long-pass filtered at $650 \mathrm{~nm}$.

The quantification of signal colocalization used the Zeiss LSM510 software. Areas in confocal planes were selected so that background signals close to the skin were excluded. Areas corresponding to V-shaped boundaries of body segments also were excluded from the analysis because of the uncertainty as to whether this anatomical region represents synapses. Because of the discontinuity in pixel intensity for either signal within clusters, we determined colocalization on a pixel-by-pixel basis. The signal strengths of two fluorescence indicators were determined individually so that pixels were deemed to be colocalized only when both crossed threshold. The thresholds for determination of colocalization of both fluorescent signals were set to one-half of the strongest signal amplitude encountered in each selected area. Colocalization of SV2 and rapsyn was performed with two protocols. First, colocalization was determined for large regions of body segments that included both Btx- positive and Btx-negative muscle in the rescued sop fish. These broad areas typically corresponded to hemisegments (see Fig. $5 \mathrm{~A}$ ) and included $\sim 30,000-100,000$ pixels, depending on the resolution of the image. Second, analyses of Btx-positive clusters were performed by restricting colocalization measurements to $50 \mu \mathrm{m}^{2}$ areas encircling SV2 and rapsyn clusters with a circle (see Fig. $5 B$ ). The clusters averaged $\sim 10-100$ pixels compared with the 500-1500 pixels of the region of analysis. The fraction of $\mathrm{X}(+) \mathrm{Y}(+)$ pixels among $\mathrm{Y}(+)$ pixels was calculated for each area and used to determine the extent of colocalization and for statistical computations. To compare the colocalization of signals between fish (see Fig. 5), we relied on statistical comparisons of selected areas, not pixels, using the Student's $t$ test.

Transient expression of sop. The mouse transgene for rescue of sop was constructed by ligating in sequence: $\alpha$-actin promoter sequence (Higashijima et al., 1997), Kozak sequence, AChR $\beta$ or $\delta$ subunit mouse cDNA, and polyadenylation signal sequence. The vector was derived from pEGFP-N1 of Clontech (Palo Alto, CA). The injection of DNA into one-cell-stage embryos was performed as described previously (Ono et al., 2002). The construct for expression of the zebrafish $\delta$ subunit cDNA in sop was similar to the murine $\beta / \delta$ clones except that, in addition, an EGFP cDNA driven by the cytomegalovirus (CMV) promoter was included. The $\delta$ subunit sequence used in the construct was amplified with PCR from wild-type fish cDNA and sequenced. The $\delta$ subunit with L28P mutation was amplified with PCR from cDNA of sop fish. The cDNA was sequenced to make sure that it harbored only the L28P mutation. Constructs were linearized at a $\mathrm{XhoI}$ site before injection into one-cell-stage sop embryos (Fig. 2). For the rescue experiments of sop shown in Figure 4 in which SV2, AChR, and rapsyn-GFP were visualized, the clone without GFP gene was used.

\section{Results}

\section{sop is an AChR $\delta$ subunit gene mutant}

Previous studies on sop revealed an absence of labeling by either $\alpha$-bungarotoxin fluorescent derivatives or $\alpha$ subunit-specific antibodies and an absence of both spontaneous and evoked synaptic responses. It was concluded from these findings that AChRs were missing at the neuromuscular junction of sop, rendering the homozygous fish immotile (Ono et al., 2001). If the mutation was in one of the five different AChR subunits, only the $\beta$ or $\delta$ subunits were candidates. An $\alpha$ subunit mutation can be ruled out because sop fish complement nic- 1 fish, which are known to carry a mutation on the $\alpha$ subunit (Sepich et al., 1998). The $\gamma$ and $\epsilon$ subunits are unlikely candidates because one can substitute for the other in permitting assembly of the receptor (Missias et al., 1997; Takahashi et al., 2002). In contrast, the $\beta$ subunit is essential for assembly and function of muscle nicotinic receptors in all species (Liu and Brehm, 1993; Paradiso and Brehm, 1998). The $\delta$ subunit is not required for assembly and function in mammals (Kullberg et al., 1990), but it is required for expression of frog AChRs (Paradiso and Brehm, 1998). Therefore, the genes coding for the $\beta$ and $\delta$ subunits were the primary candidates we set out to test.

Initially, the murine $\beta$ and $\delta$ subunits were expressed individually in sop fish in an attempt to rescue AChR expression in muscle. For this purpose DNA encoding either subunit was microinjected into the one-cell stage of eggs generated from incrossing of heterozygous sop parents. Expression was driven by the fish muscle $\alpha$-actin promoter, which effectively and specifically targets foreign proteins in zebrafish muscle (Higashijima et al., 1997; Ono et al., 2002). At $4 \mathrm{~d}$ after injection the embryos were screened for any sign of behavioral rescue. Noninjected homozygous sop fish are incapable of either touch-induced or spontaneous movements. Additionally, noninjected homozygous sop fish exhibit a complete lack of labeling by rh- $\alpha$-Btx or AChR-specific antibodies (Ono et al., 2001). Similar results were obtained from fish injected with the murine $\beta$ subunit. One-quarter of the fish was completely immotile, with no labeling by rh- $\alpha$-Btx detected 


\section{A}

Xenopus Human

Rat

Torpedo

Zebrafish

sofa potato

\section{1}

MAWIWISLLL PILIYFPGCF SESEEERLLN MEGPVLTLGL LAALAVCGSW GLNEEERLIR MAGPVPTLGL LAALVVCGSW GLNEEQRLIQ MGNIHFVYLL ISCLYYSGCS GVNEEERLIN MMKTVLLTAS LLCFIFOECC GRNEEERLIN MMKTVLLTAS LLCFIFQECC GRNEEERPIN

$*$

Signal sequence

B

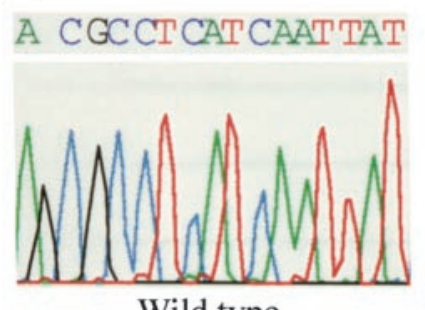

Wild type
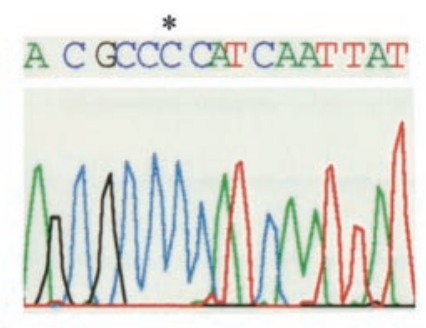

sop

Figure 1. A, Amino acid sequence alignment of $A C h R \delta$ subunit genes. The 30 amino acids starting from the first methionine are shown for sequences from zebrafish, Xenopus, human, rat, and Torpedo. Conserved amino acids among all species are boxed and shaded. The 28th amino acid is mutated from $L$ to $P$ in sop (indicated with an asterisk). The signal sequence deleted in the final form is indicated. $B$, Sequence data from wild type (left) and sop (right). T to C change in sop sequence is indicated with an asterisk.

(data not shown). In contrast, one-quarter of the fish injected with the $\delta$ subunit gene showed signs of voluntary twitching and weak swimming in response to touch. In these poor swimmers rh- $\alpha$-Btx staining was detected in some muscle cells along the ends as well as at punctate sites in the middle of muscle cells, reminiscent of AChR distribution in wild-type fish. This finding pointed to the $\delta$ subunit as the culprit mutated gene.

To identify the mutation, we cloned $\delta$ subunit cDNA from zebrafish. Zebrafish $\delta$ has $66 \%$ amino acid identity with murine $\delta$. Comparison of the sequences for wild-type and sop $\delta$ revealed a single amino acid difference. The 28th amino acid, corresponding to the sixth amino acid following the end of the signal peptide, was changed from leucine to proline in sop. This leucine is conserved in $\delta$ subunit genes of all reported species, suggesting the functional importance of the amino acid in this position (Fig. 1).

To test directly whether this single amino acid change was responsible for the phenotype of sop, we expressed individually in sop fish the wild-type and mutant $\delta$ subunit constructs containing a CMV-EGFP cassette (Fig. 2). After several days of development the muscle cells expressing the introduced $\delta$ gene under control of the $\alpha$-actin promoter were identified by green fluorescence. When wild-type fish $\delta$ subunit DNA was injected into embryos from a heterozygous sop fish in-cross, one-quarter of the embryos showed weak motility patterns similar to those injected with the murine $\delta$ subunit DNA (Fig. 2A). Fish injected with L28P-mutated $\delta$ subunit DNA showed no movement in response to touch (Fig. $2 B$ ). Confocal analysis of these fish revealed almost wild-type levels of rh- $\alpha$-Btx staining in muscle cells expressing GFP in the cytoplasm (Fig. $2 A$ ). The pattern of rh- $\alpha$-Btx distribution was also similar to that of wild-type fish. In the cells lacking GFP expression, on the other hand, rh- $\alpha$-Btx labeling was nonexistent or minimal. When cDNA coding for fish $\delta$ subunit containing the L28P mutation was injected into sop fish, onequarter of the embryos was completely immotile. These fish subsequently were screened for green fluorescence in muscle and
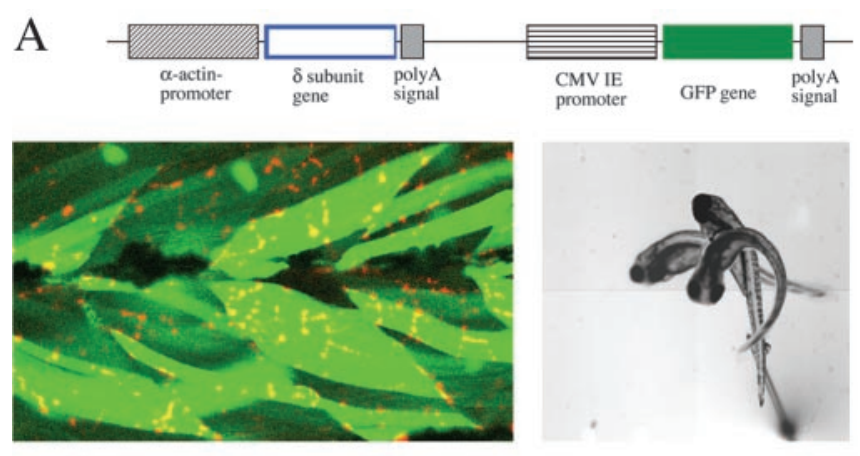

B
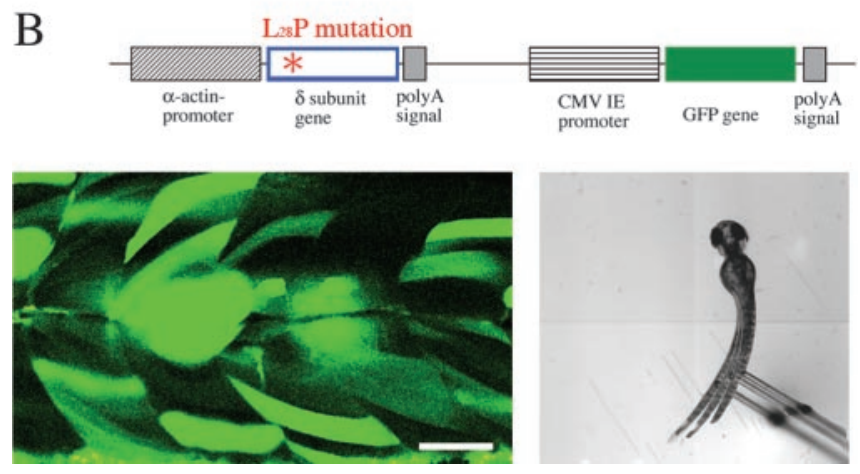

Figure 2. Rescue of sop phenotype with introduction of zebrafish $\delta$ subunit gene. The DNA constructs used for injection of wild-type $(A)$ and L28P-mutated $(B) \delta$ subunit are indicated above each corresponding fluorescence image. Each construct contains two genes under two different promoters: the $\delta$ subunit gene under $\alpha$-actin promoter and the GFP gene under CMV promoter. Shown is fluorescence imaging of tail muscle from a 4-d-old fish injected at the single-cell stage with either wild-type $\delta$ subunit $(A)$ or L28P mutant $\delta$ subunit ( $B)$. The GFP-positive muscle cells (green) injected with wild-type $\delta$ subunit exhibit associated labeling by rh-conjugated $\alpha$-bungarotoxin $(A)$, whereas this label is absent in the fish injected with mutant $\delta$ subunit $(B)$. Superimposed high speed images of fish swimming in response to touch are shown for both $A$ and $B$. Fish injected with wild-type $\delta$ subunit can mount an escape response $(A)$, whereas no movement is detected in fish injected with mutant $\delta$ subunit $(B)$. Scale bar, $50 \mu \mathrm{m}$.

then labeled with $\mathrm{rh}-\alpha$-Btx. In five fish that were examined, no labeling by rh- $\alpha$-Btx could be detected by confocal microscopy. Even in those muscle cells with the highest expression of GFP we failed to detect any rh- $\alpha$-Btx-associated fluorescence (Fig. $2 B$ ). These findings show that the L28P mutation in the AChR $\delta$ subunit gene mutation is responsible for the blocked expression of AChRs in sop.

\section{Subsynaptic distributions of rapsyn and AChRs determined by SV2 labeling}

Having established the identity of the sop mutation, we next examined the spatial relationship between postsynaptic rapsyn clusters and presynaptic nerve terminals. For this purpose we used an antibody directed against SV2, a vesicular protein involved in presynaptic exocytosis (Buckley and Kelly, 1985). This method effectively labeled presynaptic nerve terminals when coupled to a Cy5-conjugated secondary antibody. Rapsyn-GFP distribution was visualized simultaneously by means of a transgenic sop line that expresses the protein under control of a muscle-specific promoter (Ono et al., 2001). In wild-type transgenic fish carrying a single copy of rapsyn-GFP the majority of rapsyn-GFP molecules localizes to subsynaptic sites. This approach provides for simultaneous measurements of colocalization by rapsyn and motor nerve terminals. Additionally, in both wild-type fish and rescued sop fish the colocalization of AChR and rapsyn can be determined by labeling of receptors with rh- 
$\alpha$-Btx. The fluorescence labeling by SV2 antibody, however, was not as robust as that observed for rh- $\alpha$-Btx, so the pseudocolor for rh- $\alpha$-Btx was converted from the red used in Figure 2 to blue for Figures 3-5. This increased the color contrast so that spatial comparisons were easier to discern.

We first obtained day 3 wild-type fish that were carrying a single copy of the rapsyn-GFP transgene. Fish carrying a single copy of the rapsyn-GFP transgene were considerably dimmer under stereofluorescence than those with two copies. The former fish were fixed, treated with rh- $\alpha$-Btx to label the receptors, and then subsequently labeled with the anti-SV2 antibody and the secondary antibody with Cy5 to label nerve terminals (Fig. 3, wild type). Confocal imaging clearly revealed a consistent association between SV2 and rh- $\alpha$-Btx label. Synapses revealed by the colocalization of SV 2 and rh- $\alpha$-Btx always had associated clusters of rapsyn-GFP. Because of the colocalization of the three signals, synapses are shown as white spots in the merged image. sop fish showed a dramatically different pattern (Fig. 3, sop). Because this fish lacks AChR expression, only SV2 and rapsyn-GFP could be visualized. The nerve endings labeled by SV2 exhibit a weak visual overlap with the rapsyn-GFP signal. To quantify the association between SV2 label and rapsyn-GFP, we correlated the distribution of each label on a pixel-by-pixel basis. A pixel was deemed positive for either GFP or Cy5 when the signal intensity was $>50 \%$ of the strongest signal in the confocal slice. Because SV2 staining typically labels the skin as well and the imaging is done with the skin intact, it was important to exclude areas close to the skin from our analysis. In wild-type fish the value for SV2 $(+)$ and rapsyn-GFP $(+)$ pixels among SV2 $(+)$ pixels is $25.5 \pm 12.8 \%$ (13 hemisegments in 3 fish). The corresponding value for sop is $2 \pm$ $2 \%$ (14 hemisegments in 3 fish; Fig. 5A). This quantitation confirmed our finding that synaptic localization of rapsyn clusters was compromised in sop.

\section{Rapsyn-AChR distribution in rescued muscle of sop}

A direct role for AChRs in conferring subsynaptic localization of rapsyn was tested by introducing the fish $\mathrm{AChR} \delta$ subunit into sop one-cell-stage embryos. For these experiments the EGFP coding cassette was deleted from the vector because the cytosolic GFP signal would obscure the rapsyn-GFP signal. After $3 \mathrm{~d}$ of development the candidates for rescued fish were identified on the basis of weak swimming and spontaneous twitching. Only one of the parents harbored the rapsyn-GFP transgene, ensuring that fluorescent embryos had, at most, a single copy of rapsyn-GFP. Labeling candidate fish with $\mathrm{rh}-\alpha$-Btx showed discrete clusters at positions on muscle that are expected to correspond to synapses: along the edges of myocomata and at punctate sites in the middle of muscle (Fig. 4). Visual comparisons of the physical proximities of the SV2, rh- $\alpha$-Btx, and rapsyn-GFP label suggested that significant colocalization existed in muscle cells expressing the wildtype fish $\delta$ subunit (Fig. 4). Importantly, the majority of muscle cells in all candidate fish failed to exhibit rh- $\alpha$-Btx labeling, confirming the identification of $s o p^{-I-}$ on the basis of behavior.

Two different methods were undertaken to quantitate the ability of receptor expression to rescue the subsynaptic localization of rapsyn-GFP observed in rescued muscle as seen in Figure 4. First, estimates of colocalization of rapsyn-GFP clusters and nerve terminals were determined for large regions on the basis of SV2-positive pixels that also exhibited rapsyn-GFP label (Fig. $5 A)$. The measurements included an area of muscle that exhibited at least one $\mathrm{rh}-\alpha-\mathrm{Btx}(+)$ muscle cell but also included additional regions containing muscle cells that were SV2 $(+)$ but rh- $\alpha$ $\operatorname{Btx}(-)$. In this analysis the percentage of pixels above threshold for SV2 $(+)$ or rapsyn-GFP is $<1 \%$, because the majority of pixels in the area is close to the background level and deemed negative. The value for SV2 $(+)$ and rapsyn-GFP $(+)$ double positive pixels among SV2 $(+)$ pixels measured $13 \pm 11 \%$ (9 hemisegments in 3 fish). This value obtained for rescued sop fish was significantly higher than $2 \pm 2 \%$ obtained for $\operatorname{sop}$ ( $p<0.01$, Student's $t$ test). However, it was significantly lower than $25.5 \pm 12.8 \%$ obtained for wild type ( $p<0.01$, Student's $t$ test; Fig. $5 A$ ). This difference between wild-type fish and the rescued sop fish stems presumably from the mosaic expression of the wild-type $\delta$ subunit gene in the rescued fish wherein only a subpopulation of muscle cells expresses AChRs on the cell surface (Fig. $2 A$ ). Indeed, the percentage of $\operatorname{Btx}(+) \operatorname{SV} 2(+)$ double positive pixels among SV2 $(+)$ pixels is $38 \pm 17 \%$ in wild-type fish, whereas it is $13 \pm 8 \%$ in the rescued sop fish.

In a second series of measurements the analyses were re- 

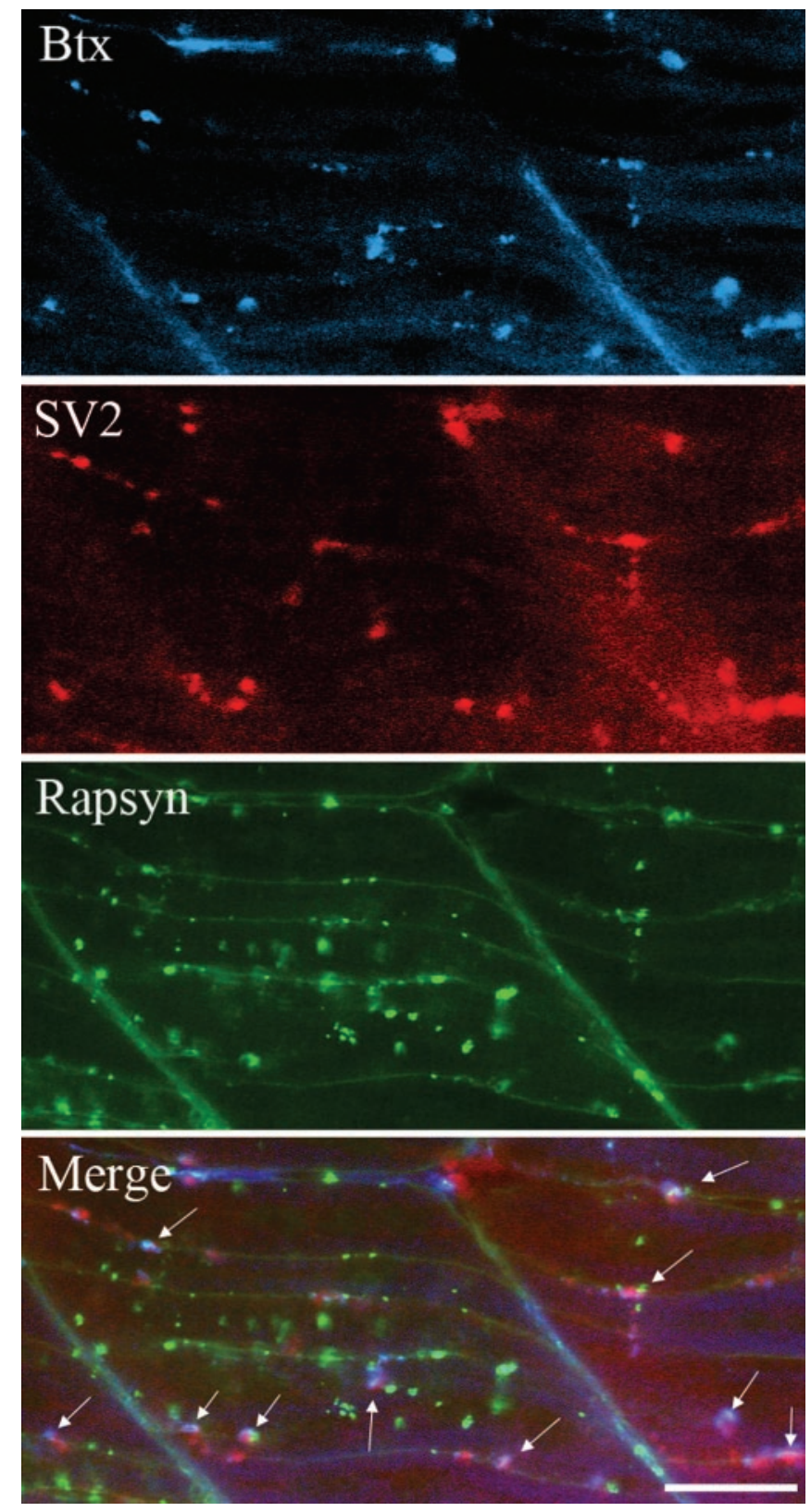

Figure 4. Synaptic localization of rapsyn-GFP is rescued in sop muscle cells expressing wildtype $\delta$ subunit gene. A $72 \mathrm{hr}$ sop fish that was injected with wild-type $\delta$ subunit at the singlecell stage shows wild-type-like labeling by rh-conjugated $\alpha$-bungarotoxin in some muscle cells (Btx panel). The position of nerve terminals is indicated by anti-SV2 (SV2 panel) and the location of rapsyn by GFP (Rapsyn panel). The relative location of all three indicators is indicated by the merged image (Merge panel). Examples of locations that exhibit overlap of three signals are indicated with arrows. Scale bar, $20 \mu \mathrm{m}$.

stricted to rh- $\alpha$-Btx-positive regions within single muscle cells of rescued sop fish (Fig. $5 B$ ). A $50 \mu \mathrm{m}^{2}$ circular area was circumscribed around identified $\operatorname{rh}-\alpha-\mathrm{Btx}(+)$ clusters. The circumscribed area was, in all cases, considerably larger than the rh- $\alpha$ Btx cluster. In this analysis of the restricted area the percentage of pixels above threshold for SV2 $(+)$ or rapsyn-GFP ranged from 2 to $15 \%$. Among these $\mathrm{rh}-\alpha-\mathrm{Btx}(+)$ sites a measure of colocalization between nerve terminals and rapsyn-GFP was provided by calculating the percentage of SV2 $(+)$ and rapsyn-GFP $(+)$ double positive pixels among SV2 $(+)$ pixels. At these receptorpositive sites colocalization coefficients corresponded to $48 \pm$ $21 \%$ for wild type and $43 \pm 18 \%$ for rescued $\operatorname{sop}(p>0.05$,
A

B
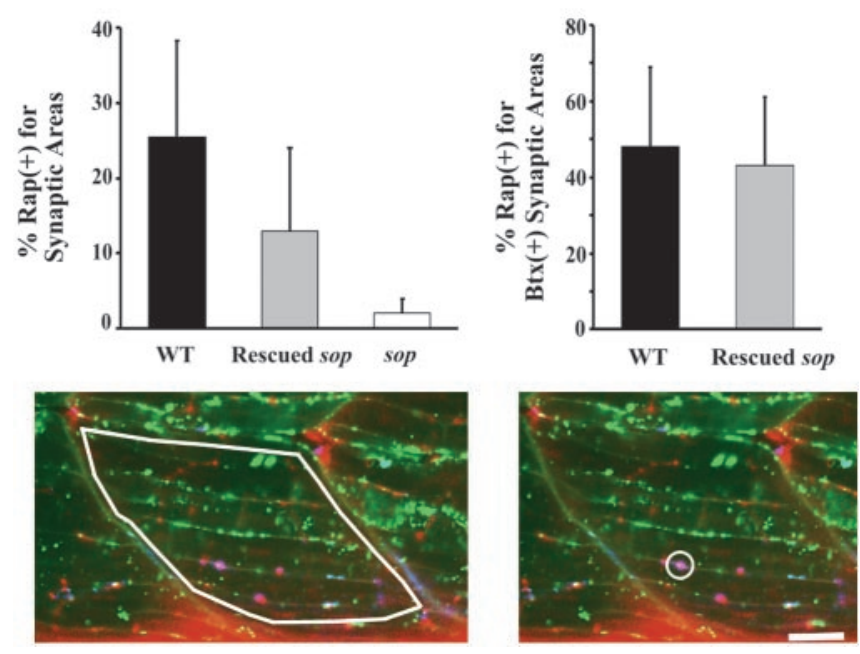

Figure 5. Quantitation of rapsyn localization to synaptic sites in wild type, sop, and rescued sop. $A$, The percentage of SV2(+) pixels that colocalize with rapsyn-GFP $(+)$ pixels within a large region corresponding to multiple muscle cells. In the case of the rescued fish this area included regions of muscle that were not expressing receptors. $B$, Similar measurements from synaptic sites in muscle identified as expressing receptors on the basis of Btx labeling. In the areas judged to represent Btx clusters, the percentage of rapsyn-GFP $(+)$ pixels among SV2(+) pixels is shown. Mean \pm SDs are indicated. Typical areas in rescued sop fish used to calculate the colocalization for the two methods of analyses are delineated with white lines below each graph. In $A$, a ventral or dorsal half of a body segment was used. Note that Btx signal is detected only in some muscle cells. In $B$, an area encompassing a synapse expressing AChRs judged by $\operatorname{Btx}(+)$ signal is used. Scale bar, $20 \mu \mathrm{m}$.

Student's $t$ test; Fig. $5 B$ ). By random occurrence the colocalization would be expected to be no larger than $2 \%$. This estimate is based on the largest size clusters observed for both signals. In the data shown in Figure $5 \mathrm{~A}$ and for smaller cluster sizes, the probability of random colocalization would be much smaller. These data indicate that rapsyn more effectively localizes to subsynaptic membrane when associated with AChRs.

\section{Discussion}

Our results demonstrate that in sop fish an L28P mutation in the coding region of the $\delta$ subunit gene completely abolishes the expression of AChRs on the muscle cell surface. We find no evidence of AChR expression in this mutant line on the bases of either synaptic recordings or labeling with rh- $\alpha$-Btx or $\alpha$ subunit antibody (Ono et al., 2001). Consequently, sop fish are paralyzed completely and incapable of any behavioral responses to touch. Expression of this mutated $\delta$ subunit in sop fish fails to restore swimming or expression of surface AChRs. However, transient expression of either murine or zebrafish wild-type $\delta$ subunit in sop fish is able to partially restore swimming movement and surface expression of the AChRs in muscle.

Curiously, in another mutant zebrafish line also lacking expression of AChRs, nic-1, the homozygous fish are able to mount slow twitching movements of their tail (Sepich et al., 1998). Such movements have never been observed at any stage of development in sop fish. The source of this movement is not clear, but it is possible that nic-1 is not completely receptor null despite the absence of labeling by rh- $\alpha$-Btx. In nic- 1 the lack of surface receptors results from a mutation in the $\alpha$ subunit AChR gene, but the mutation resides in an intron rather than in an exon, leaving open the possibility that a small number of $\alpha$ subunits potentially could be transcribed and assembled into AChRs. Alternatively, a 
second muscle type $\alpha$ subunit, recently discovered in the gene family of pufferfish, could account for low-level expression of receptors in nic-1 (Jones et al., 2003). Thus, although some question remains as to whether nic- 1 is a true null allele, there seems to be no question about sop. To date, no equivalent AChR null lines have been reported for mammals, presumably because of the lethal consequences of a total knock-out. Both the $\gamma$ and $\epsilon$ subunits of the AChR subunits have been knocked out individually in mice, but neither represents an AChR null line because these two subunits are able to substitute for one another during development (Missias et al., 1997; Takahashi et al., 2002). As a result, knock-out of either subunit will only prevent expression of the adult or embryonic form of the receptor. Consequently, zebrafish mutant lines provide a unique vertebrate system in which neuromuscular development can be studied in the absence of AChRs (Westerfield et al., 1990; Ono et al., 2001; Li et al., 2003).

The initial characterization of sop provided new insights into the individual roles of rapsyn and AChRs during synapse development. For example, studies on both cultured muscle and heterologous expression systems reached different conclusions as to whether rapsyn can self-cluster in the absence of receptor. When rapsyn was expressed in fibroblasts (Phillips et al., 1991) or in Xenopus oocytes (Froehner et al., 1990), rapsyn formed selfclusters without the coexpression of AChRs. On the other hand, in C2 myotubes lacking expression of AChRs, the stability of rapsyn was decreased and agrin treatment failed to cause aggregation of rapsyn (Marangi et al., 2001). Our results from in vivo showed that rapsyn was capable of self-clustering in the absence of receptor. More significant, however, was the observation that rapsyn clusters show greatly reduced ability to localize to the synapse in the absence of receptor (Ono et al., 2001). This led to the intriguing proposition that AChRs were not merely escorted to the synaptic sites by their partner rapsyn but that instead the AChRs might play an active role in localizing rapsyn clusters to the subsynaptic membrane. This idea was fueled further by findings from another neuromuscular mutant line, twitch once (Ono et al., 2002). In twitch once, rapsyn is mutated in one of tetratricopeptide repeats (TPRs). The mutated rapsyn retained the ability to form self-clusters, but AChR clusters failed to form, suggesting that rapsyn-AChR interaction was disrupted by this mutation. An inability of rapsyn clusters to localize to the synapse in this mutant line provided further support for the proposition that association with AChRs was required for proper navigation. We now confirm a role for the AChR in directing rapsyn clusters to the synapse through rescue of sop. In muscle cells wherein the fish $\delta$ subunit is expressing at levels permissive to formation of fully assembled AChRs, we find significantly higher levels of AChR-rapsyn clusters at synaptic sites. Moreover, the mutant $\delta$ subunit is unable to rescue AChR expression, showing that the mutation is directly responsible for the inability of rapsyn clusters to undergo synaptic localization.

The sites governing rapsyn-receptor interaction were investigated first by using heterologous cells. These studies indicate that the coiled-coil regions of rapsyn mediate receptor interaction (Ramarao and Cohen, 1998; Ramarao et al., 2001). However, in vivo studies on a mutant line of zebrafish have indicated that the tetratricopeptide region of rapsyn is involved. A single mutation in the fourth tetratricopeptide repeat annihilates interaction with receptor (Ono et al., 2002). The mechanisms causal to the dependence of rapsyn on the AChR for synaptic localization are less clear. One possibility is that the receptor provides a direct anchor for the rapsyn cluster. Without the anchor the rapsyn clusters are free to move about the cell surface. In fact, the association be- tween synaptic MuSK (muscle-specific kinase) and rapsyn requires an as yet to be identified transmembrane linker (Apel et al., 1997). This function could be served by the transmembrane AChR. Alternatively, the receptor-dependent synaptic localization of rapsyn may not be mediated directly by the AChR. Instead, receptors might be required for the formation of a larger synaptic complex that serves as a prerequisite for anchoring. A different role for the AChR in rapsyn navigation is suggested on the bases of recent studies showing that rapsyn and receptor are cotransported and inserted into the plasma membrane (Marchand et al., 2000, 2002). The lack of receptor in the complex could abrogate insertion and lead to ineffective assembly at the subsynaptic sites.

The effects of mutations in the extreme N-terminal region of the $\delta$ subunit on AChR expression and/or function have not been examined. However, according to the structure of a homologous protein AChBP (Brejc et al., 2001), the 28th leucine is in the first extracellular $\alpha$ helix and might be expected to affect folding of the $\delta$ subunit. Thus the mutated $\delta$ subunit might be unable to associate properly with the other subunits of the AChR. Given that other zebrafish neuromuscular mutants have increased our understanding of human neuromuscular diseases such as myasthenic syndrome (Ohno et al., 2002; Ono et al., 2002) and slow channel syndrome (Engel et al., 2003; Lefebvre et al., 2004), it seems important to consider the potential implications of a $\delta$ subunit null in the human population. In frog the $\delta$ subunit is absolutely required for functional expression of both embryonic and adult-type AChRs (Paradiso and Brehm, 1998), and our findings from sop indicate that a similar dependence exists for fish. However, unlike fish and frogs, the $\delta$ subunit is apparently not essential for surface expression of embryonic-type AChRs in mammals (Kullberg and Brehm, 1990; Liu and Brehm, 1993). This species difference raises the interesting possibility that mutations resulting in omission of the $\delta$ subunit may be viable in the human population. What is the expected phenotype of $\delta$-less receptors in vivo? Because the $\delta$ subunit is required for adult receptors ( $\epsilon$-containing), but not embryonic ( $\gamma$-containing) receptors, knocking out $\delta$ expression would be similar to an $\epsilon$ knock-out. Curiously, no $\epsilon$ knock-out fish have been identified to date, despite the fact that this subunit is the most highly mutated subunit in the human population. However, in both mouse knock-out (Witzemann et al., 1996) and human syndromes lacking $\epsilon$ (Engel et al., 1996; Milone et al., 1998; Croxen et al., 1999), the consequence is muscle weakness very early in life, worsening with age. This is because the normal transition to adult AChR kinetics does not occur in these animals, and the developmental elimination of embryonic receptors is delayed in the absence of $\epsilon$ (Missias et al., 1997). Thus our findings on sop predict a potentially different behavioral outcome for an equivalent mutation in human $\delta$. A sop equivalent in the human population may exist and be causal to undisclosed and rare forms of neuromuscular disorders.

\section{References}

Apel ED, Glass DJ, Moscoso LM, Yancopoulos GD, Sanes JR (1997) Rapsyn is required for MuSK signaling and recruits synaptic components to a MuSK-containing scaffold. Neuron 18:623-635.

Behra M, Cousin X, Bertrand C, Vonesch JL, Biellmann D, Chatonnet A, Strahle U (2002) Acetylcholinesterase is required for neuronal and muscular development in the zebrafish embryo. Nat Neurosci 5:111-118.

Brejc K, van Dijk WJ, Klaassen RV, Schuurmans M, van der Oost J, Smit AB, Sixma TK (2001) Crystal structure of an ACh-binding protein reveals the ligand-binding domain of nicotinic receptors. Nature 411:269-276.

Buckley K, Kelly RB (1985) Identification of a transmembrane glycoprotein 
specific for secretory vesicles of neural and endocrine cells. J Cell Biol 100:1284-1294.

Croxen R, Newland C, Betty M, Vincent A, Newsom-Davis J, Beeson D (1999) Novel functional $\epsilon$-subunit polypeptide generated by a single nucleotide deletion in acetylcholine receptor deficiency congenital myasthenic syndrome. Ann Neurol 46:639-647.

Engel AG, Ohno K, Bouzat C, Sine SM, Griggs RC (1996) End-plate acetylcholine receptor deficiency due to nonsense mutations in the epsilon subunit. Ann Neurol 40:810-817.

Engel AG, Ohno K, Sine SM (2003) Congenital myasthenic syndromes: progress over the past decade. Muscle Nerve 27:4-25.

Froehner SC, Luetje CW, Scotland PB, Patrick J (1990) The postsynaptic $43 \mathrm{~K}$ protein clusters muscle nicotinic acetylcholine receptors in Xenopus oocytes. Neuron 5:403-410.

Granato M, van Eeden FJ, Schach U, Trowe T, Brand M, Furutani-Seiki M, Haffter P, Hammerschmidt M, Heisenberg CP, Jiang YJ, Kane DA, Kelsh RN, Mullins MC, Odenthal J, Nüsslein-Volhard C (1996) Genes controlling and mediating locomotion behavior of the zebrafish embryo and larva. Development 123:399-413.

Higashijima S, Okamoto H, Ueno N, Hotta Y, Eguchi G (1997) Highfrequency generation of transgenic zebrafish which reliably express GFP in whole muscles or the whole body by using promoters of zebrafish origin. Dev Biol 192:289-299.

Jones AK, Elgar G, Sattelle DB (2003) The nicotinic acetylcholine receptor gene family of the pufferfish, Fugu rubripes. Genomics 82:441-451.

Kullberg R, Owens JL, Camacho P, Mandel G, Brehm P (1990) Multiple conductance classes of mouse nicotinic acetylcholine receptors expressed in Xenopus oocytes. Proc Natl Acad Sci USA 87:2067-2071.

Lefebvre JL, Ono F, Puglielli C, Saidner G, Franzini-Armstrong C, Brehm P, Granato M (2004) Increased neuromuscular activity causes axonal defects and muscle degeneration. Development 31:2605-2618.

Li W, Ono F, Brehm P (2003) Optical measurements of presynaptic release in mutant zebrafish lacking postsynaptic receptors. J Neurosci 23:10467-10474.

Liu Y, Brehm P (1993) Expression of subunit-omitted mouse nicotinic acetylcholine receptors in Xenopus laevis oocytes. J Physiol (Lond) 470:349-363.

Marangi PA, Forsayeth JR, Mittaud P, Erb-Vogtli S, Blake DJ, Moransard M, Sander A, Fuhrer C (2001) Acetylcholine receptors are required for agrininduced clustering of postsynaptic proteins. EMBO J 20:7060-7073.

Marchand S, Bignami F, Stetzkowski-Marden F, Cartaud J (2000) The myristoylated protein rapsyn is cotargeted with the nicotinic acetylcholine receptor to the postsynaptic membrane via the exocytic pathway. J Neurosci 20:521-528.

Marchand S, Devillers-Thiery A, Pons S, Changeux JP, Cartaud J (2002) Rapsyn escorts the nicotinic acetylcholine receptor along the exocytic pathway via association with lipid rafts. J Neurosci 22:8891-8901.
Milone M, Ohno K, Fukudome T, Shen XM, Brengman J, Griggs RC, Engel AG (1998) Congenital myasthenic syndrome caused by novel loss-offunction mutations in the human AChR epsilon subunit gene. Ann NY Acad Sci 841:184-188.

Missias AC, Mudd J, Cunningham JM, Steinbach JH, Merlie JP, Sanes JR (1997) Deficient development and maintenance of postsynaptic specializations in mutant mice lacking an "adult" acetylcholine receptor subunit. Development 124:5075-5086.

Ohno K, Engel AG, Shen XM, Selcen D, Brengman J, Harper CM, Tsujino A, Milone M (2002) Rapsyn mutations in humans cause endplate acetylcholine-receptor deficiency and myasthenic syndrome. Am J Hum Genet 70:875-885.

Ono F, Higashijima S, Shcherbatko A, Fetcho J, Brehm P (2001) Paralytic zebrafish lacking acetylcholine receptors fail to localize rapsyn clusters to the synapse. J Neurosci 21:5439-5448.

Ono F, Shcherbatko A, Higashijima S, Mandel G, Brehm P (2002) The zebrafish motility mutant twitch once reveals new roles for rapsyn in synaptic function. J Neurosci 22:6491-6498.

Paradiso K, Brehm P (1998) Long-term desensitization of nicotinic acetylcholine receptors is regulated via protein kinase A-mediated phosphorylation. J Neurosci 18:9227-9237.

Phillips WD, Kopta C, Blount P, Gardner PD, Steinbach JH, Merlie JP (1991) ACh receptor-rich membrane domains organized in fibroblasts by recombinant 43-kildalton protein. Science 251:568-570.

Ramarao MK, Cohen JB (1998) Mechanism of nicotinic acetylcholine receptor cluster formation by rapsyn. Proc Natl Acad Sci USA 95:4007-4012.

Ramarao MK, Bianchetta MJ, Lanken J, Cohen JB (2001) Role of rapsyn tetratricopeptide repeat and coiled-coil domains in self-association and nicotinic acetylcholine receptor clustering. J Biol Chem 276:7475-7483.

Sanes JR, Lichtman JW (2001) Induction, assembly, maturation and maintenance of a postsynaptic apparatus. Nat Rev Neurosci 2:791-805.

Sepich DS, Wegner J, O'Shea S, Westerfield M (1998) An altered intron inhibits synthesis of the acetylcholine receptor $\alpha$-subunit in the paralyzed zebrafish mutant nic1. Genetics 148:361-372.

Takahashi M, Kubo T, Mizoguchi A, Carlson CG, Endo K, Ohnishi K (2002) Spontaneous muscle action potentials fail to develop without fetal-type acetylcholine receptors. EMBO Rep 3:674-681.

Westerfield M, Liu DW, Kimmel CB, Walker C (1990) Pathfinding and synapse formation in a zebrafish mutant lacking functional acetylcholine receptors. Neuron 4:867-874.

Witzemann V, Schwarz H, Koenen M, Berberich C, Villarroel A, Wernig A, Brenner HR, Sakmann B (1996) Acetylcholine receptor $\epsilon$-subunit deletion causes muscle weakness and atrophy in juvenile and adult mice. Proc Natl Acad Sci USA 93:13286-13291. 